\title{
Age and Changes in Extracted Features of Lagged Poincare Plot
}

\author{
Shahab Rezaei ${ }^{1}$, Sadaf Moharreri ${ }^{2}$, Nader Jafarnia Dabanloo ${ }^{3}$, Saman Parvaneh ${ }^{4}$ \\ ${ }^{1}$ Islamic Azad University, Central Tehran Branch, Tehran, Iran \\ ${ }^{2}$ Islamic Azad University, Khomeini Shahr Branch, Isfahan, Iran \\ ${ }^{3}$ Islamic Azad University, Science and Research Branch, Tehran, Iran \\ ${ }^{4}$ Philips Research North America, Cambridge, MA, USA
}

\begin{abstract}
The Poincare plot is a geometrical representation of $R R$ time series constructed by plotting successive $R R$ intervals on a $2 D$ phase space. In this article, the impact of age on the shape of Poincare plot of RR intervals and extracted features for quantification of this space is considered. Fantasia database from Physionet databank is used in this paper. Two hours of ECG recording (sampling frequency: $250 \mathrm{~Hz}$ ) for twenty young (21-34 years old) and twenty older adults (68-85 years old) were used while all subjects remained in a resting state. After extraction of RR intervals from ECG, Poincare plot with 10 different lags (1-10) were constructed for each $R R$ series, and eleven different features were extracted for each lag. Extracted features from lagged Poincare plot were used as input to K-nearest neighbor classifier to discriminate between two groups of young and older adults. Sensitivity of $86.5 \%$, specificity of $95.1 \%$, and the accuracy of $91.4 \%$ was achieved in the classification.
\end{abstract}

\section{Introduction}

Physiological responses such as heart rate and heart rate variability (HRV) change by age and frailty [1]. Previous studies examined the influence of age on the various indexes used for quantification of HRV [2]. Research has shown that aging is associated with a significant decline in HRV features [3, 4]. As the age increases, the heart rate slows down and the blood vessels lose their elasticity to some extent and become tighter. By tightening the arteries, the heart works harder to pump blood into the veins this leads to an increase in blood pressure and other cardiovascular problems in the elderly [2]. Therefore, recognizing the effects of age on cardiac parameters can be useful in preventing many diseases.

The HRV is changes in the time intervals between consecutive heartbeats over time that shows heart adaptation to accept peripheral changes [5]. Analysis of the Poincare plot is a very powerful visual technique that detects hidden patterns in the HRV and provides summary information about the entire RR interval time series [6]. The plot has two dimensions and each point consists of a pair of successive intervals. Research show that using longer lags have useful information for capturing different information from HRV signal [7].

Furthermore, in recent years different features for quantification of Poincare plot of RR intervals have been introduced and showed promising results in emotion recognition $[8,9]$ and arrhythmia detection $[10,11]$.

In this paper, Poincare plot with 10 different lags were constructed, and various features were extracted in each lagged plot. The obtained features were used to evaluate the impact of age on heart behavior as well as for classification of the two groups of young and older subjects.

\section{Data and Method}

\subsection{Data}

For analyzing the impact of age on HRV, Fantasia database from Physionet databank was used [12]. Two hours of ECG recording for 20 young (21-34 years old) and 20 older adults (68-85 years old) subjects were used while all subjects remained in a resting state. The sampling frequency for this database is $250 \mathrm{~Hz}$.

\subsection{Method}

\subsubsection{QRS Detection}

The Pan-Tompkins algorithm was used for QRS detection [13]. As suggested in the guideline [14], the output of the QRS detection algorithm visually checked by an expert and any ectopic beats were excluded. Finally, RR interval series were created using corrected QRS peaks. 


\subsubsection{Lagged Poincare Plot}

Given $R R$ time series as $\left(R_{1}, R_{2}, \ldots, R R_{n}\right)$, the lagged Poincare plot is constructed by plotting points $p_{i}\left(x_{i}, y_{i}\right)$ in which $x$ and $y$ are defined as:

$$
\begin{gathered}
x=\left\{R R_{1}, R R_{2}, R R_{3, \ldots,} R R_{n-\text { lag }}\right\} \\
y=\left\{R R_{1+\text { lag }}, R R_{2+\text { lag }}, R R_{3+\text { lag }, \ldots,},\right. \\
\left.\quad R R_{n+\text { lag }}\right)
\end{gathered}
$$

in which lag show the value of delay in lagged Poincare plot.

\subsubsection{Standard Descriptors of Poincare Plot}

$S D 1$ and SD2 are defined as the standard deviation of projection of the points in Poincare plot on the line perpendicular to the identity line $(y=-x)$ and identity line $(y=x)$, respectively [15].

\subsubsection{Complex Correlation Measure (CCM)}

Complex Correlation Measure (CCM) introduced by Karmakar et al. for quantifying temporal variation in points' distribution in Poincare plot [16]. By considering every three consecutive points $a\left(x_{i}, y_{i}\right), b\left(x_{i+1}, y_{i+1}\right)$, and $c\left(x_{i+2}, y_{i+3}\right)$, the following determinant is computed [16]:

$$
A(i)=\frac{1}{2}\left|\begin{array}{ccc}
x_{i} & y_{i} & 1 \\
x_{i+1} & y_{i+1} & 1 \\
x_{i+2} & y_{i+2} & 1
\end{array}\right|
$$

And so CCM is defined as [16]:

$$
C C M(m)=\frac{1}{C_{n}(\mathrm{n}-2)} \sum_{i=1}^{n-2} A(i)
$$

where $m$ represents lag of Poincare plot and $C_{n}$ is the normalizing constant which is defined as [16]:

$$
C_{n}=\pi * S D 1 * S D 2
$$

\subsubsection{Global Occurrence Matrix (GOM)}

For measuring the distribution of points relative to the identity line $(y=x)$, Moharreri et al. proposed GOM

\begin{tabular}{|c|c|c|c|c|c|c|c|c|c|c|c|c|}
\hline \multirow{2}{*}{ Lag } & \multirow{2}{*}{ Groups } & \multicolumn{11}{|c|}{ Extracted Features } \\
\hline & & $S D I$ & $S D 2$ & $S D 1 / S D 2$ & $C C M$ & $\mathrm{Cn}$ & $N_{A}$ & $N_{O}$ & $N_{B}$ & $A$ & B & C \\
\hline \multirow{2}{*}{1} & Young & $0.12 \pm 0.03$ & $0.17 \pm 0.03$ & $0.54 \pm 0.06$ & $-0.20 \pm 0.005$ & $0.16 \pm 0.15$ & $1344 \pm 1090$ & $105 \pm 56$ & $1355 \pm 79$ & $0.44 \pm 0.07$ & $-1.98 \pm 0.83$ & $1.56 \pm 0.41$ \\
\hline & Old & $0.05 \pm 0.002$ & $0.08 \pm 0.002$ & $0.59 \pm 0.09$ & $-0.19 \pm 0.007$ & $0.02 \pm 0.0007$ & $1273 \pm 1027$ & $251 \pm 132$ & $1266 \pm 77$ & $0.34 \pm 0.06$ & $-0.74 \pm 0.54$ & $0.41 \pm 0.10$ \\
\hline \multirow{2}{*}{2} & Young & $0.13 \pm 0.03$ & $0.17 \pm 0.03$ & $0.65 \pm 0.03$ & $0.06 \pm 0.005$ & $0.16 \pm 0.15$ & $1416 \pm 251$ & $77 \pm 37$ & $1311 \pm 46$ & $0.27 \pm 0.04$ & $-0.82 \pm 0.21$ & $0.60 \pm 0.56$ \\
\hline & Old & $0.05 \pm 0.001$ & $0.09 \pm 0.002$ & $0.59 \pm 0.04$ & $0.09 \pm 0.007$ & $0.02 \pm 0.0008$ & $1284 \pm 680$ & $202 \pm 112$ & $1302 \pm 62$ & $0.17 \pm 0.05$ & $-0.36 \pm 0.50$ & $0.20 \pm 0.08$ \\
\hline \multirow{2}{*}{3} & Young & $0.13 \pm 0.03$ & $0.17 \pm 0.03$ & $0.65 \pm 0.03$ & $0.02 \pm 0.002$ & $0.16 \pm 0.15$ & $1411 \pm 476$ & $86 \pm 56$ & $1306 \pm 44$ & $0.23 \pm 0.04$ & $-0.76 \pm 0.40$ & $0.57 \pm 0.01$ \\
\hline & Old & $0.06 \pm 0.002$ & $0.09 \pm 0.002$ & $0.60 \pm 0.03$ & $-0.002 \pm 0.001$ & $0.02 \pm 0.0008$ & $1282 \pm 579$ & $206 \pm 102$ & $1300 \pm 57$ & $0.14 \pm 0.04$ & $-0.28 \pm 0.45$ & $0.15 \pm 0.08$ \\
\hline \multirow{2}{*}{4} & Young & $0.13 \pm 0.03$ & $0.17 \pm 0.03$ & $0.69 \pm 0.02$ & $-0.02 \pm 0.001$ & $0.16 \pm 0.15$ & $1393 \pm 185$ & $76 \pm 41$ & $1333 \pm 36$ & $0.20 \pm 0.02$ & $-0.68 \pm 0.24$ & $0.53 \pm 0.65$ \\
\hline & Old & $0.06 \pm 0.001$ & $0.09 \pm 0.002$ & $0.63 \pm 0.03$ & $-0.005 \pm 0.0005$ & $0.02 \pm 0.0007$ & $1307 \pm 382$ & $174 \pm 78$ & $1304 \pm 58$ & $0.13 \pm 0.03$ & $-0.30 \pm 0.36$ & $0.18 \pm 0.04$ \\
\hline \multirow{2}{*}{5} & Young & $0.14 \pm 0.03$ & $0.16 \pm 0.03$ & $0.74 \pm 0.02$ & $-0.003 \pm 0.001$ & $0.17 \pm 0.15$ & $1388 \pm 148$ & $60 \pm 28$ & $1352 \pm 31$ & $0.15 \pm 0.07$ & $-0.50 \pm 0.80$ & $0.40 \pm 0.73$ \\
\hline & Old & $0.06 \pm 0.002$ & $0.09 \pm 0.002$ & $0.66 \pm 0.03$ & $-0.004 \pm 0.0006$ & $0.02 \pm 0.0008$ & $1317 \pm 305$ & $152 \pm 72$ & $1316 \pm 52$ & $0.11 \pm 0.02$ & $-0.25 \pm 0.30$ & $0.15 \pm 0.03$ \\
\hline \multirow{2}{*}{6} & Young & $0.14 \pm 0.03$ & $0.16 \pm 0.03$ & $0.75 \pm 0.02$ & $0.005 \pm 0.001$ & $0.17 \pm 0.15$ & $1374 \pm 176$ & $70 \pm 40$ & $1356 \pm 34$ & $0.14 \pm 0.07$ & $-0.46 \pm 0.85$ & $0.37 \pm 0.81$ \\
\hline & Old & $0.06 \pm 0.002$ & $0.09 \pm 0.002$ & $0.69 \pm 0.02$ & $0.003 \pm 0.0003$ & $0.02 \pm 0.0008$ & $1320 \pm 214$ & $151 \pm 68$ & $1313 \pm 48$ & $0.09 \pm 0.02$ & $-0.21 \pm 0.27$ & $0.13 \pm 0.02$ \\
\hline \multirow{2}{*}{7} & Young & $0.14 \pm 0.03$ & $0.16 \pm 0.03$ & $0.75 \pm 0.02$ & $0.009 \pm 0.002$ & $0.17 \pm 0.15$ & $1368 \pm 129$ & $66 \pm 33$ & $1365 \pm 38$ & $0.14 \pm 0.06$ & $-0.56 \pm 0.25$ & $0.46 \pm 0.65$ \\
\hline & Old & $0.06 \pm 0.002$ & $0.08 \pm 0.002$ & $0.70 \pm 0.02$ & $0.002 \pm 0.0005$ & $0.02 \pm 0.0008$ & $1325 \pm 185$ & $137 \pm 59$ & $1321 \pm 48$ & $0.09 \pm 0.01$ & $-0.20 \pm 0.24$ & $0.12 \pm 0.02$ \\
\hline \multirow{2}{*}{8} & Young & $0.13 \pm 0.03$ & $0.16 \pm 0.03$ & $0.73 \pm 0.01$ & $0.001 \pm 0.0003$ & $0.17 \pm 0.15$ & $1361 \pm 98$ & $65 \pm 31$ & $1372 \pm 37$ & $0.16 \pm 0.03$ & $-1.45 \pm 0.29$ & $1.30 \pm 0.26$ \\
\hline & Old & $0.06 \pm 0.002$ & $0.08 \pm 0.002$ & $0.72 \pm 0.02$ & $-0.001 \pm 0.001$ & $0.02 \pm 0.0008$ & $1326 \pm 226$ & $130 \pm 56$ & $1327 \pm 49$ & $0.08 \pm 0.01$ & $-0.18 \pm 0.22$ & $0.11 \pm 0.01$ \\
\hline \multirow{2}{*}{9} & Young & $0.14 \pm 0.03$ & $0.16 \pm 0.03$ & $0.76 \pm 0.01$ & $-0.006 \pm 0.001$ & $0.17 \pm 0.15$ & $1353 \pm 123$ & $68 \pm 32$ & $1376 \pm 38$ & $0.16 \pm 0.04$ & $-1.85 \pm 0.16$ & $1.70 \pm 0.49$ \\
\hline & Old & $0.06 \pm 0.002$ & $0.08 \pm 0.002$ & $0.74 \pm 0.02$ & $0.002 \pm 0.0005$ & $0.02 \pm 0.0008$ & $1324 \pm 214$ & $135 \pm 56$ & $1322 \pm 36$ & $0.07 \pm 0.01$ & $-0.16 \pm 0.20$ & $0.09 \pm 0.01$ \\
\hline \multirow[b]{2}{*}{10} & Young & $0.14 \pm 0.03$ & $0.16 \pm 0.03$ & $0.76 \pm 0.01$ & $0.003 \pm 0.001$ & $0.17 \pm 0.15$ & $1348 \pm 149$ & $68 \pm 33$ & $1380 \pm 38$ & $0.11 \pm 0.02$ & $-1.26 \pm 0.89$ & $1.17 \pm 0.41$ \\
\hline & Old & $0.06 \pm 0.002$ & $0.08 \pm 0.002$ & $0.75 \pm 0.02$ & $-0.003 \pm 0.0002$ & 0.page 2 & $1333 \pm 170$ & $123 \pm 54$ & $1324 \pm 44$ & $0.05 \pm 0.01$ & $-0.13 \pm 0.17$ & $0.08 \pm 0.01$ \\
\hline
\end{tabular}
features which are the number of points above $\left(N_{A}\right)$, on $\left(N_{O}\right)$, and below $\left(N_{B}\right)$ the identity line [10]:

$$
\mathrm{GOM}=\left[\mathrm{N}_{\mathrm{A}} \mathrm{N}_{\mathrm{O}} \mathrm{N}_{\mathrm{B}}\right]
$$

Table 1. Mean and standard deviation of extracted features for each lag of Poincare plot for young and old groups 
Table 2. $p$-value of extracted features for each lag of Poincare plot for young and old groups

\begin{tabular}{|c|c|c|c|c|c|c|c|c|c|c|c|}
\cline { 2 - 12 } \multicolumn{1}{c|}{} & \multicolumn{10}{c|}{ Extracted Features } \\
\hline Lag & SD1 & SD2 & SD1/SD2 & CCM & Cn & $\mathbf{N}_{\mathbf{A}}$ & No & $\mathbf{N}_{\mathbf{B}}$ & A & B & C \\
\hline $\mathbf{1}$ & 0.137 & 0.020 & 0.725 & 0.552 & 0.083 & 0.034 & 0.033 & 0.001 & 0.194 & 0.213 & 0.159 \\
\hline $\mathbf{2}$ & 0.035 & 0.048 & 0.372 & 0.245 & 0.051 & $<0.001$ & 0.011 & 0.978 & 0.074 & 0.055 & 0.030 \\
\hline $\mathbf{3}$ & 0.055 & 0.037 & 0.317 & 0.040 & 0.058 & $<0.001$ & 0.026 & 0.850 & 0.066 & 0.070 & 0.105 \\
\hline $\mathbf{4}$ & 0.079 & 0.042 & 0.256 & 0.499 & 0.051 & $<0.001$ & 0.046 & 0.144 & 0.058 & 0.083 & 0.066 \\
\hline $\mathbf{5}$ & 0.045 & 0.040 & 0.130 & 0.705 & 0.042 & $<0.001$ & 0.005 & 0.021 & 0.110 & 0.105 & 0.123 \\
\hline $\mathbf{6}$ & 0.058 & 0.035 & 0.223 & 0.291 & 0.051 & $<0.001$ & 0.059 & 0.006 & 0.152 & 0.130 & 0.051 \\
\hline $\mathbf{7}$ & 0.048 & 0.033 & 0.344 & 0.176 & 0.048 & $<0.001$ & 0.062 & 0.006 & 0.079 & 0.130 & 0.066 \\
\hline $\mathbf{8}$ & 0.079 & 0.040 & 0.978 & 0.570 & 0.055 & $<0.001$ & 0.233 & 0.002 & 0.093 & 0.117 & 0.062 \\
\hline $\mathbf{9}$ & 0.083 & 0.033 & 0.935 & 0.433 & 0.055 & 0.026 & 0.169 & $<0.001$ & 0.099 & 0.137 & 0.055 \\
\hline $\mathbf{1 0}$ & 0.093 & 0.023 & 0.685 & 0.358 & 0.055 & 0.213 & 0.757 & $<0.001$ & 0.185 & 0.317 & 0.152 \\
\hline
\end{tabular}

\subsubsection{Parabolic Phase Space Mapping (PPSM)}

Jafarnia et. al. introduced a new phase space which consist the points as follows [17]:

$$
\left(x,(\overline{R R}-y)^{2}\right)
$$

In which $\overline{R R}$ is the mean of $R R$ intervals that is defined as:

$$
\operatorname{mean}(R R)=\overline{R R}=\frac{1}{n+1} \sum_{i=1}^{n} R R_{i}
$$

A two degree polynomial equation in the form of $\mathrm{Y}=$ $A \mathrm{x}^{2}+B \mathrm{x}+C$ has been estimated for the points distribution, in which [11]:

$$
Y=(\overline{R R}-y)^{2}
$$

And the coefficients of the estimated polynomial $(A, B$, and $C$ ) are the extracted features in this phase space [17]

\subsubsection{Statistical Analysis and Classification}

Mean and standard deviation were reported for all extracted features in each lag in Table 1. To identify features that are significantly different in two groups of young and old groups, Kruskal-Wallis test was used with the level of significance set to 0.05 .

K-nearest neighbor (KNN) classifier was used to classify two groups of young and old that was trained on $70 \%$ of data as a train set, and the accuracy was evaluated on $30 \%$ of data as a test set.

\section{Results and Discussion}

As it is shown in Table 1, the obtained results of comparing extracted features of Poincare plot in two groups of young and old people, prove the results in previous studies, extracted features decrease when the age grows $[3,4]$.

The results of Kruskal-Wallis test are shown in Table 2. The results show that SD2 and the three parameters of GOM were significantly different between two groups. The selected features which had significant $p$-value were used as an input for $k N N$ classifier and the two groups of young and old have been considered as an output of the classifier. The classifier performance on test set is shown in Table 3.

Table 3. Classification performance on test data

\begin{tabular}{ccc}
\hline Accuracy & Sensitivity & Specificity \\
\hline $91.4 \%$ & $86.5 \%$ & $95.1 \%$ \\
\hline
\end{tabular}

\section{Conclusion}

In this article, extracted features from lagged Poincare plot of RR intervals were compared between two groups of young and old subjects to evaluate the impact of age on HRV Parameters. These features were then used as an input for K-nearest neighbor classifier. The results show that the novel features specially GOM which represent the dynamic behavior of RR intervals is a useful measure for studying the impact age on cardiac autonomic nervous system.

\section{References}

[1] S. Parvaneh et al., "Regulation of cardiac autonomic nervous system control across frailty statuses: a systematic review," Gerontology, vol. 62 (1), pp. 3-15, 2016.

[2] M. W. Agelink, B. Baumann, T. Majewski, F. Akila, T. Zeit, and D. Ziegler, "Standardized tests of heart rate variability: normal ranges obtained from 309 healthy humans, and effects of age, gender, and heart rate," Clinical Autonomic Research, vol. 11, pp. 99-108, 2001.

[3] S. Rukavina, S. Gruss, H. Hoffmann, J.-W. Tan, S. Walter, 
and H. C. Traue, "Affective computing and the impact of gender and age," PloS one, vol. 11, no. 3, 2016.

[4] L. Burriss, D. A. Powell, and J. White, "Psychophysiological and subjective indices of emotion as a function of age and gender," Cognition \& Emotion, vol. 21 (1), pp. 182-210, 2007.

[5] C. K. Karmakar, A. Khandoker, and M. Palaniswami, "Analysis of slope based heart rate asymmetry using poincare plots," presented at the Computing in Cardiology (CinC), 2012.

[6] M. Karimi Moridani, S. K. Setarehdan, A. M. Nasrabadi, and E. Hajinasrollah, "Non-Linear feature extraction from hrv signal for mortality prediction of icu cardiovascular patient," Journal of Medical Engineering \& Technology, vol. 40 (3), pp. 87-98, 2016.

[7] C. Lerma, O. Infante, H. Perez-Grovas, and M. V. Jose, "Poincare plot indexes of heart rate variability capture dynamic adaptations after haemodialysis in chronic renal failure patients," Clinical Physiology and Functional Imaging, vol. 23 (2), pp. 72-80, 2003.

[8] S. Moharreri, N. Jafarnia Dabanloo, S. Parvaneh, and A. M. Nasrabadi, "The relation between colors, emotions and heart response using triangle phase space mapping (TPSM)," presented at the Computing in Cardiology (CinC2011), 2011.

[9] S. Moharreri, N. Jafarnia Dabanloo, S. Parvaneh, and A. M. Nasrabadi, "How to interpret psychology from heart rate variability?," in Middle East Conference on Biomedical Engineering (MECBME), Sharjah, UAE, 2011, vol. 1: IEEE.

[10] S. Moharreri, S. Parvaneh, N. Jafarnia Dabanloo, and A. M. Nasrabadi, "Utilizing occurrence sequence of heart rate's phase space points in order to discriminate heart arrhythmia," in the 17th Iranian Conference of Biomedical Engineering (ICBME2010), Isfahan, Iran, 2010, vol. 17: IEEE.

[11] N. Jafarnia Dabanloo, S. Moharreri, S. Parvaneh, and A. M. Nasrabadi, "New representation of heart rate and evaluation of extracted geometric features," presented at the Computing in Cardiology (CinC), Belfast, Northern Ireland, 2010.

[12] A. L. Goldberger et al., "Physiobank, physiotoolkit, and physionet components of a new research resource for complex physiologic signals," Circulation, vol. 101, no. 23, pp. e215e220, 2000.

[13] J. Pan and W. J. Tompkins, "A real-time QRS detection algorithm," Biomedical Engineering, IEEE Transactions on, no. 3, pp. 230-236, 1985.

[14] M. Malik, "Heart rate variability," Annals of Noninvasive Electrocardiology, vol. 1, no. 2, pp. 151-181, 1996.

[15] J. Piskorski and P. Guzik, "Filtering Poincaré plots," Computational methods in science and technology, vol. 11, no. 1, pp. 39-48, 2005.

[16] C. K. Karmakar, A. H. Khandoker, J. Gubbi, and M. Palaniswami, "Complex correlation measure: a novel descriptor for poincare plot," Biomedical Engineering OnLine, vol. 8, pp. $1-12,2009$.

[17] N. Jafarnia Dabanloo, S. Moharreri, S. Parvaneh, and A. M. Nasrabadi, "Application of novel mapping for heart rate phase space and its role in cardiac arrhythmia diagnosis," presented at the Computing in Cardiology (CinC 2010), 2010.

Address for correspondence.

Saman Parvaneh

2 Canal Park, 3rd floor, Cambridge, MA 02141

parvaneh@ieee.org 